\title{
Demographic trends among older cannabis users in the United States, 2006-13
}

\author{
Benjamin H. Han ${ }^{1,2}$, Scott Sherman ${ }^{1,2,3}$, Pia M. Mauro ${ }^{4}$, Silvia S. Martins ${ }^{4}$, James Rotenberg' \& \\ Joseph J. Palamar ${ }^{2,3}$
}

Department of Medicine, Division of Geriatric Medicine and Palliative Care, New York University School of Medicine, New York, NY, USA,' Center for Drug Use and HIV Research, New York University College of Nursing, New York, NY, USA, ${ }^{2}$ Department of Population Health, New York University School of Medicine, New York, NY, $U_{S A}^{3}$ and Department of Epidemiology, Mailman School of Public Health, Columbia University, New York, NY, USA ${ }^{4}$

\section{ABSTRACT}

Background and Aims The ageing US population is providing an unprecedented population of older adults who use recreational drugs. We aimed to estimate the trends in the prevalence of past-year use of cannabis, describe the patterns and attitudes and determine correlates of cannabis use by adults age 50 years and older. Design Secondary analysis of the National Survey on Drug Use and Health survey from 2006 to 2013, a cross-sectional survey given to a nationally representative probability sample of populations living in US households. Setting USA. Participants A total of 47140 survey respondents aged $\geq 50$ years. Measures Estimates and trends of past-year use of cannabis. Findings The prevalence of past-year cannabis use among adults aged $\geq 50$ increased significantly from 2006/07 to 2012/13, with a $57.8 \%$ relative increase for adults aged 50-64 (linear trend $P<0.001$ ) and a 250\% relative increase for those aged $\geq 65$ (linear trend $P=0.002$ ). When combining data from 2006 to $2013,6.9 \%$ of older cannabis users met criteria for cannabis abuse or dependence, and the majority of the sample reported perceiving no risk or slight risk associated with monthly cannabis use $(85.3 \%)$ or weekly use (79\%). Past-year users were more likely to be younger, male, nonHispanic, not have multiple chronic conditions and use tobacco, alcohol or other drugs compared with non-past-year cannabis users. Conclusions The prevalence of cannabis use has increased significantly in recent years among US adults aged $\geq 50$ years.

Keywords Aging, cannabis, epidemiology, geriatrics, older adult, substance use.

Correspondence to: Benjamin H. Han, New York University School of Medicine, Department of Medicine, Division of Geriatric Medicine and Palliative Care, 550 First Avenue, BCD 615, New York, NY 10016, USA. E-mail: benjamin.han@nyumc.org

Submitted 12 February 2016; initial review completed 17 June 2016; final version accepted 19 October 2016

\section{INTRODUCTION}

The United States is experiencing sweeping changes in attitudes towards cannabis ('marijuana') use and associated policy. Recreational cannabis use was legalized in 2012 in Colorado and Washington states [1,2], with additional states decriminalizing its use and/or allowing the prescribing of medical cannabis [3]. This is occurring in the setting of a rapidly aging population, with the large Baby Boomer generation (born between 1946 and 1964) reaching 65 years of age in 2011 [4]. The Baby Boomer generation has higher reported rates of substance use compared to any generation preceding it, and stark differences in attitudes towards recreational and illicit drug use [5-7]. However, there is limited research that addresses the epidemiology and health status of older adults who use drugs. We also understand little about how drug use impacts aging or how aging influences substance use. The lack of knowledge in this area constrains the care for a changing demographic of older adults with higher rates of substance use.

Cannabis is not only one of the most commonly used drugs in the United States [8], western and central Europe and Oceania [9]; it is also the most prevalent drug after alcohol and tobacco used by adults aged 50 and older in the United States and the United Kingdom [5,10]. A recent study using the National Epidemiologic Survey on Alcohol and Related Conditions showed that the prevalence of past-year cannabis use more than doubled from 2001-02 to 2012-13 [11], with large increases among those aged 45-64 years. Another recent study using age-period-cohort analysis found that recent increases in 
past-year cannabis use are not unique to younger generations [12].

However, only a handful of studies have examined cannabis use among older adults. Studies show that the majority of older adults who use cannabis started their use as adolescents or as young adults [13]. Prevalence of past-month cannabis use among older adults with depression who sought mental health treatment was found to be $12 \%$ for men and $4 \%$ for women [14]. A cross-sectional study of the 1999-2001 National Household Surveys on Drug Abuse showed that lower education level was correlated with past-year cannabis use by adults aged 50 and older [15] and analysis of the 2008 National Survey on Drug use and Health (NSDUH) showed that among middle-aged and older adults, cannabis users were more likely to be younger, black and more likely to smoke cigarettes and/or use other substances [16]. Another recent study using the 2012-13 National Epidemiologic Survey on Alcohol and Related Conditions (NESARC-III) found that among adults aged 50 and older, 3.9\% reported past-year cannabis use and $21.7 \%$ reported being former users, and both past-year and former users had high risk for mental disorders [17]. However, more studies examining current trends over time and correlations of cannabis use by older adults are lacking.

Amid recent changes in attitudes towards cannabis and increasing support for legalization [18-20], coupled with the continued aging of the Baby Boomer generation and the potential use of cannabis to cope with aging and chronic disease side effects [21,22], cannabis use by older adults may continue to increase. A large international survey on the medicinal use of cannabis conducted by the International Association for Cannabinoid Medicine in 31 countries found that $30.5 \%$ of the 953 participants surveyed were over the age of 50 [23]. The survey also found that the top five indications for cannabis use included chronic pain, anxiety, loss of appetite or weight loss, depression or sleeping disorder, all common conditions in older age. While an abundance of research has focused upon cannabis as a risk factor for neurocognitive abnormalities in the developing brain of adolescents [24,25], there may be distinctive risks of cannabis use by older adults such as cognitive impairment or cardiovascular risk [6], and these are not well understood. Further studies are needed to determine changes in the characteristics of older adults who use cannabis, and what potential risks and benefits it may pose.

In summary, while we recognize that cannabis use may be increasing among older adults, recent trends in prevalence of cannabis use and patterns, attitudes and correlates of its use by middle-aged and older adults are lacking. With the aim of filling key gaps in the current literature, the present study aims to: [1] estimate the prevalence of past-year cannabis use by older adults from 2006 to 2013; [2] report on the trends in past-year cannabis use by older adults from 2006 to 2013; [3] describe patterns and attitudes regarding cannabis use among past-year older users during the study period; and [4] determine correlates of past-year cannabis use among older adults. To address these questions, the current study used cross-sectional data from eight years (2006-13) of a nationally representative sample of non-institutionalized individuals in the United States.

\section{METHODS}

\section{Data source and study population}

Data were obtained from the last eight cohorts (2006-13) of NSDUH, an ongoing cross-sectional survey of noninstitutionalized individuals in the 50 US states and the District of Columbia [26], with different participants sampled each year and therefore independent of each other. NSDUH is a nationally representative probability sample of populations living in households obtained through four stages. Census tracts were first selected within each state, then segments in each tract, then dwelling, and then respondents were selected for the final sample each year.

Surveys were administered via computer-assisted interviewing (CAI) conducted by an interviewer and audio computer-assisted self-interviewing (ACASI). Sampling weights were provided by NSDUH to address unit- and individual-level non-response. Weights were adjusted to ensure that estimates were consistent with estimates provided by the US Census Bureau. Further information on sampling and survey techniques can be found elsewhere [26]. The weighted interview response rates for 2006-13 NSDUH ranged from 71.7 to $75.7 \%$.

\section{Measures}

Participants were asked whether they had used 'marijuana' within the last 12 months. They were also asked whether they had used tobacco, alcohol or illicit drugs other than 'marijuana'. Participants were asked about their perception of risk of using 'marijuana' once a month and answer options were 'no risk', 'slight risk', 'moderate risk' and 'great risk'. The sample was also asked about perception of risk of using 'marijuana' once or twice a week and they were provided with the same answer options. Those who reported cannabis use were also asked some follow-up questions about use. Participants answered a series of questions that determined whether criteria would meet Diagnostic and Statistical Manual of Mental Disorders, 4th edition (DSM-IV) abuse or dependence for cannabis. While not a diagnostic interview, this provided a proxy diagnosis. Participants were also asked their age of cannabis initiation and frequency of use during the last 12 months. Those reporting use were also asked about their method of obtaining 'marijuana' the last time they 
used it and answer options were 'bought', 'traded for it', 'received it for free/shared', 'grew it' and 'unspecified'. Similarly, those reporting use were asked about the source of their most recent 'marijuana' purchase and answer options were 'bought it from a friend', 'relative/family', 'someone they just met or did not know' and 'unspecified'.

We examined subject age (predefined as ageds 50-64 and $\geq 65$ ), gender, race (i.e. white non-Hispanic, black non-Hispanic, Hispanic, other race), educational attainment (i.e. $<$ high school, high school, some college, college or more), annual total income (i.e. $<$ \$20 000; \$20 00049 999; \$50 000-74999; \$75000+) and marital status (i.e. married, widowed, divorced or separated or never married).

Participants were asked if they were ever told by a doctor or other medical professional that they have had any of the following chronic diseases: diabetes, heart disease, stroke, sleep apnea or cirrhosis. We recoded the responses into a binary variable indicating multiple chronic conditions ('yes' indicating that the participant reported two or more of the listed chronic diseases and 'no' signifying having fewer than two of the chronic diseases). Participants were also asked whether they had experienced depression or anxiety within the last year. They were also asked whether had received mental health treatment within the last year.

\section{Statistical analyses}

To achieve the aims of this study, we focused on individuals aged $\geq 50$, who represented approximately $22-25 \%$ of the full sample each year. As self-reported cannabis use was relatively rare, we aggregated years into pairs to increase power to detect trends over time. Specifically, similar to previous analyses [11,27], we collapsed years into 2006-07, 2008-09, 2010-11 and 2012-13.

We first examined descriptive statistics to estimate the weighted prevalence of self-reported past-year use of cannabis across cohorts, as well as the prevalence of past-year use of cannabis separately for each category of each covariate. We computed the absolute change over time by subtracting prevalence of cannabis use in 2012/13 from that in 2006/07. We also computed the relative change over time by dividing the prevalence of cannabis use in $2012 / 13$ by that in 2006/07. We then estimated whether there was a linear association between cannabis use and time within each of the covariate categories; statistical significance was assessed at the two-sided $\alpha<0.05$ level. We further estimated weighted prevalences of the patterns and attitudes regarding cannabis use among past-year selfreported cannabis users aged 50 and older, aggregating the sample across all years from 2006 to 2013. In particular, we estimated overall weighted prevalence of DSM-IV cannabis abuse or dependence, age of first use, frequency and method of obtaining cannabis, as well as perceived risk associated with cannabis use.

We then estimated odds of cannabis use over time and by socio-demographic, substance use, and health covariates in the full sample using logistic regression. Separate bivariable models were used to determine associations between each covariate and cannabis use, which produced unadjusted odds ratios (ORs), and a multivariable model was computed to examine the association of all covariates with all else being equal. This produced an adjusted OR (aOR) for each level of each covariate. We conducted sensitivity analyses testing two- and three-way interactions between age, gender and time to assess, for example, whether the adjusted odds of cannabis use over time differed between older and younger females. Analyses were weighted to account for the complex survey design. As our analyses used data from eight NSDUH years, we divided the survey weights by 8 to obtain nationally representative estimates. Data were analyzed using Stata SE version 13 (StataCorp, College Station, TX, USA; 2009), which used the Taylor series estimation methods to provide accurate standard errors [28]. This secondary data analysis of publically available data was exempt for review by the New York University Langone Medical Center Institutional Review Board.

\section{RESULTS}

Sample characteristics across cohorts are presented in Table 1. The prevalence of past-year cannabis use among adults aged 50 and older increased significantly from $2006 / 07$ to $2012 / 13$ (Table 2). The prevalence in $2006 / 07$ increased significantly to $2012 / 13$ from 2.8 to $4.8 \%(P<0.001)$, and this was a $71.4 \%$ relative increase. Older adults aged $\geq 65$ had a significantly lower prevalence of cannabis use compared to adults aged 50-64 (i.e. 1.4 versus $7.1 \%$ in 2012/13); however, ages 65+ had a larger relative increase $(250 \%)$ than adults aged 50-64 (57.8\%).

Prevalence of past-year cannabis use was higher among men than women throughout all years, and in 2012/13 the prevalence of past-year cannabis use among men was twice as high as the prevalence among women over the age of 50 (6.8 versus 3.0\%). The prevalence of self-reported past-year cannabis use was increased significantly by $76 \%$ among non-Hispanic white participants from 2006/07 to 2012/13 ( $P<0.001)$. Past-year cannabis use increased significantly from 2006/07 to 2012/13 among individuals across all levels of education ( $P s<0.05)$, with incomes below $\$ 50000(P s<0.01)$ and those who were married $(P<0.001)$, divorced or separated $(P<0.05)$.

The 2012/13 prevalence of past-year cannabis use among reported past-year illicit (other than 'marijuana') drug users was $34.8 \%$, a relative increase of $16.8 \%$ since 
Table 1 Sample characteristics across cohorts of adults aged 50 and older, \% (n).

\begin{tabular}{|c|c|c|c|c|c|}
\hline & $\begin{array}{l}2006 / 2007 \\
(n=11181)\end{array}$ & $\begin{array}{l}2008 / 2009 \\
(n=10891)\end{array}$ & $\begin{array}{l}2010 / 2011 \\
(n=12372)\end{array}$ & $\begin{array}{l}2012 / 2013 \\
(n=12696)\end{array}$ & $\begin{array}{l}\text { Combined years } \\
(n=47140)\end{array}$ \\
\hline Prevalence of cannabis use & 2.8 & 3.3 & 4.1 & 4.8 & 3.8 \\
\hline \multicolumn{6}{|l|}{ Age group (years, \%) } \\
\hline $50-64$ & $59.4(6904)$ & $59.5(6778)$ & $59.6(7742)$ & $58.7(7859)$ & $59.3(29283)$ \\
\hline $65+$ & $40.6(4277)$ & $40.5(4113)$ & $40.4(4630)$ & $41.3(4837)$ & $40.7(17857)$ \\
\hline \multicolumn{6}{|l|}{ Gender } \\
\hline Male & $46.0(5044)$ & $46.3(4912)$ & $46.5(5585)$ & $46.8(5698)$ & $46.4(21239)$ \\
\hline Female & $54.0(6137)$ & $53.7(5979)$ & $53.5(6787)$ & $53.2(6998)$ & $53.6(25901)$ \\
\hline \multicolumn{6}{|l|}{ Race/ethnicity } \\
\hline Non-Hispanic White & $77.5(8813)$ & 76.7 (8297) & $76.1(9369)$ & $74.6(9400)$ & $76.2(35879)$ \\
\hline Non-Hispanic Black & $9.7(984)$ & $9.8(1045)$ & $9.8(1264)$ & $10.3(1364)$ & $9.9(4657)$ \\
\hline Hispanic & $7.9(781)$ & $8.2(909)$ & $8.7(989)$ & $9.3(1122)$ & $8.6(3801)$ \\
\hline Other & $4.9(603)$ & $5.3(640)$ & $5.5(750)$ & $5.8(810)$ & $5.4(2803)$ \\
\hline \multicolumn{6}{|l|}{ Education } \\
\hline$<$ High school & $18.3(2031)$ & $16.5(1917)$ & $15.1(1956)$ & $14.4(1981)$ & $16.0(7885)$ \\
\hline High school & $32.0(3760)$ & $33.0(3622)$ & $32.3(4094)$ & $31.6(4193)$ & $32.2(15669)$ \\
\hline Some college & $22.0(2482)$ & $22.1(2506)$ & $23.0(2934)$ & $24.0(3087)$ & $22.8(11009)$ \\
\hline College or more & $27.7(2908)$ & $28.4(2846)$ & $29.6(3388)$ & $30.0(3435)$ & $29.0(12577)$ \\
\hline \multicolumn{6}{|l|}{ Income } \\
\hline$<\$ 20000$ & $40.4(4574)$ & $38.4(4436)$ & 39.1 (4997) & $37.2(5037)$ & $38.7(19044)$ \\
\hline$\$ 20-49999$ & $35.8(4190)$ & $37.2(4078)$ & $35.0(4497)$ & $36.1(4672)$ & $36.0(17437)$ \\
\hline$\$ 50000-74999$ & $12.4(1287)$ & $12.5(1300)$ & $12.5(1468)$ & $12.5(1453)$ & $12.5(5508)$ \\
\hline$\geq \$ 75000$ & $11.4(1130)$ & $11.8(1077)$ & $13.4(1410)$ & $14.2(1534)$ & $12.8(5151)$ \\
\hline \multicolumn{6}{|l|}{ Marital status } \\
\hline Married & $63.6(7032)$ & $64.0(6687)$ & $62.2(7520)$ & $61.8(7517)$ & $62.9(28756)$ \\
\hline Widowed & $14.8(1622)$ & $13.5(1465)$ & $13.5(1646)$ & $13.0(1652)$ & $13.7(6385)$ \\
\hline Divorced or separated & $15.6(1811)$ & $15.8(1928)$ & $17.9(2307)$ & $18.5(2534)$ & $17.0(8580)$ \\
\hline Never married & $6.0(716)$ & $6.7(811)$ & $6.4(899)$ & $6.6(993)$ & $6.4(3419)$ \\
\hline \multicolumn{6}{|l|}{ Any tobacco use } \\
\hline Past year no use & $76.0(8387)$ & $76.3(8209)$ & $77.3(9322)$ & $77.6(9569)$ & $76.8(35487)$ \\
\hline Past year use & $24.0(2794)$ & $23.7(2683)$ & $22.7(3050)$ & $22.4(3127)$ & $23.2(11653)$ \\
\hline \multicolumn{6}{|l|}{ Alcohol use } \\
\hline No past-year use & 40.5 (4495) & $38.6(4300)$ & $38.7(4841)$ & $37.5(4847)$ & $38.8(18483)$ \\
\hline Past-year use & $59.5(6686)$ & $61.4(6591)$ & $61.3(7531)$ & $62.5(7849)$ & $61.2(28657)$ \\
\hline \multicolumn{6}{|c|}{ Illicit drug other than marijuana } \\
\hline No past-year use & $97.4(10903)$ & $97.2(10560)$ & $97.4(12029)$ & 96.7 (12 277) & $97.2(45769)$ \\
\hline Past-year use & $2.6(278)$ & $2.8(331)$ & $2.6(343)$ & $3.3(419)$ & $2.8(1371)$ \\
\hline \multicolumn{6}{|l|}{ Chronic disease $^{\mathrm{a}}$} \\
\hline \multicolumn{6}{|c|}{ Multiple chronic conditions ( $\geq 2$ chronic disease) } \\
\hline No & $83.5(9310)$ & $81.5(8852)$ & $81.2(10002)$ & $81.1(10321)$ & $81.8(38485)$ \\
\hline Yes & $16.5(1871)$ & $18.5(1998)$ & $18.8(2313)$ & $18.9(2375)$ & $18.2(8557)$ \\
\hline \multicolumn{6}{|l|}{ Mental health } \\
\hline \multicolumn{6}{|l|}{ Depression in the past year } \\
\hline No & $93.0(10361)$ & $92.8(10027)$ & $93.2(11417)$ & $92.4(11660)$ & $92.8(43465)$ \\
\hline Yes & $7.0(820)$ & $7.2(815)$ & $6.8(896)$ & $7.6(1036)$ & $7.2(3567)$ \\
\hline \multicolumn{6}{|l|}{ Anxiety in past year } \\
\hline No & $96.1(10727)$ & 95.4 (10 349) & 95.7 (11 748) & $95.0(11979)$ & $95.5(44803)$ \\
\hline Yes & $3.9(454)$ & $4.6(498)$ & $4.3(566)$ & $5.0(717)$ & 4.5 (2235) \\
\hline \multicolumn{6}{|c|}{ Received mental health treatment in past year } \\
\hline No & $87.3(9645)$ & 86.7 (9381) & $86.9(10622)$ & $85.2(10679)$ & 86.5 (40 345) \\
\hline Yes & 12.7 (1495) & $13.3(1473)$ & $13.1(1704)$ & $14.8(1947)$ & 13.5 (6619) \\
\hline
\end{tabular}

${ }^{\mathrm{a} C h r o n i c ~ d i s e a s e s ~ i n c l u d e ~ d i a b e t e s, ~ h e a r t ~ d i s e a s e, ~ s t r o k e, ~ s l e e p ~ a p n e a ~ a n d ~ c i r r h o s i s . ~}$

2006/07; however, this was not a significant trend ( $P=0.257$ ). The prevalence in $2012 / 13$ of past-year use of cannabis among those who reported past-year alcohol use was 6.7\%, a significant increase from 2006/07 $(P<0.001)$. Past-year cannabis use also increased significantly among past-year tobacco smokers, with a 
Table 2 Prevalence estimates for self-reported past-year cannabis use by socio demographics, chronic disease and substance use characteristics for adults 50 years and older, United States 2006-13.

\begin{tabular}{|c|c|c|c|c|c|c|c|}
\hline & $\begin{array}{l}2006 / 2007 \\
(n=11181)\end{array}$ & $\begin{array}{l}2008 / 2009 \\
(n=10891)\end{array}$ & $\begin{array}{l}2010 / 2011 \\
(n=12372)\end{array}$ & $\begin{array}{l}2012 / 2013 \\
(n=12696)\end{array}$ & $\begin{array}{l}\% \text { Absolute } \\
\text { change from } \\
2006 / 07 \text { to } \\
2012 / 13\end{array}$ & $\begin{array}{l}\% \text { Relative } \\
\text { change from } \\
2006 / 07 \text { to } \\
2012 / 13\end{array}$ & $\begin{array}{l}\text { Linear } \\
\text { trend } \\
P \text {-value }\end{array}$ \\
\hline Prevalence of cannabis use & 2.8 & 3.3 & 4.1 & 4.8 & +2.0 & +71.4 & $<0.001$ \\
\hline \multicolumn{8}{|l|}{ Age group (years) } \\
\hline $50-64$ & 4.5 & 5.2 & 6.1 & 7.1 & +2.6 & +57.8 & $<0.001$ \\
\hline $65+$ & 0.4 & 0.6 & 1.1 & 1.4 & +1.0 & +250.0 & 0.002 \\
\hline \multicolumn{8}{|l|}{ Characteristic } \\
\hline \multicolumn{8}{|l|}{ Gender } \\
\hline Male & 4.4 & 4.5 & 5.6 & 6.8 & +2.4 & +54.5 & $<0.001$ \\
\hline Female & 1.5 & 2.2 & 2.8 & 3.0 & +1.5 & +100.0 & $<0.001$ \\
\hline \multicolumn{8}{|l|}{ Race/ethnicity } \\
\hline Non-Hispanic white & 2.9 & 3.2 & 4.2 & 5.1 & +2.2 & +75.9 & $<0.001$ \\
\hline Non-Hispanic black & 3.7 & 4.8 & 5.0 & 5.3 & +1.6 & +43.2 & 0.188 \\
\hline Hispanic & 1.8 & 1.9 & 3.4 & 2.6 & +0.8 & +44.4 & 0.210 \\
\hline Other & 1.2 & 3.6 & 2.7 & 3.5 & +2.3 & +191.7 & 0.140 \\
\hline \multicolumn{8}{|l|}{ Education } \\
\hline$<$ High school & 2.5 & 2.5 & 5.1 & 5.1 & +2.6 & +104.0 & 0.008 \\
\hline High school & 2.4 & 3.4 & 3.2 & 4.6 & +2.2 & +91.7 & 0.001 \\
\hline Some college & 3.5 & 3.8 & 5.4 & 4.7 & +1.2 & +34.3 & 0.016 \\
\hline College or more & 3.0 & 3.2 & 3.6 & 5.3 & +2.3 & +76.7 & 0.003 \\
\hline \multicolumn{8}{|l|}{ Income } \\
\hline$<\$ 20000$ & 2.5 & 3.0 & 4.9 & 5.4 & +2.9 & +116.0 & $<0.001$ \\
\hline$\$ 20-49999$ & 2.5 & 3.2 & 3.4 & 4.1 & +1.6 & +64.0 & 0.002 \\
\hline$\$ 50000-74999$ & 3.7 & 3.6 & 3.9 & 4.7 & +1.0 & +27.0 & 0.360 \\
\hline$\geq \$ 75000$ & 4.1 & 4.5 & 4.0 & 4.8 & +0.7 & +17.1 & 0.636 \\
\hline \multicolumn{8}{|l|}{ Marital status } \\
\hline Married & 1.9 & 2.5 & 3.0 & 4.0 & +2.1 & +110.5 & $<0.001$ \\
\hline Widowed & 1.0 & 1.1 & 1.8 & 1.6 & +0.6 & +60.0 & 0.092 \\
\hline Divorced or separated & 6.6 & 6.5 & 8.1 & 8.5 & +1.9 & +28.8 & 0.032 \\
\hline Never married & 7.0 & 7.3 & 8.7 & 8.1 & +1.1 & +15.7 & 0.364 \\
\hline \multicolumn{8}{|l|}{ Any tobacco use } \\
\hline Past-month use & 7.4 & 9.0 & 10.6 & 12.3 & +4.9 & +66.2 & $<0.001$ \\
\hline Past-year use & 7.7 & 8.9 & 10.4 & 12.0 & +4.3 & +55.8 & $<0.001$ \\
\hline \multicolumn{8}{|l|}{ Alcohol use } \\
\hline Past-month use & 4.6 & 5.4 & 6.2 & 7.4 & +2.8 & +60.9 & $<0.001$ \\
\hline Past-year use & 4.1 & 4.9 & 5.8 & 6.7 & +2.6 & +63.4 & $<0.001$ \\
\hline Binge alcohol use in past 30 days & 8.0 & 11.0 & 11.5 & 13.4 & +5.4 & +67.5 & 0.001 \\
\hline \multicolumn{8}{|l|}{ Illicit drug other than marijuana } \\
\hline Past-month used & 28.7 & 32.8 & 29.9 & 39.3 & +10.6 & +39.9 & 0.196 \\
\hline Past-year use & 29.8 & 30.1 & 30.5 & 34.8 & +5.0 & +16.8 & 0.257 \\
\hline \multicolumn{8}{|l|}{ Chronic diseases $^{\mathrm{b}}$} \\
\hline $\begin{array}{l}\text { Multiple chronic conditions } \\
\text { ( } \geq 2 \text { chronic disease) }\end{array}$ & 1.7 & 2.2 & 3.2 & 3.6 & +1.9 & +111.8 & 0.001 \\
\hline \multicolumn{8}{|l|}{ Mental health } \\
\hline Depression in the past year & 5.7 & 4.7 & 9.3 & 11.4 & +5.7 & +100.0 & 0.001 \\
\hline Anxiety in past year & 8.2 & 4.8 & 9.5 & 9.0 & +0.8 & +9.8 & 0.247 \\
\hline $\begin{array}{l}\text { Received mental health treatment } \\
\text { in past year }\end{array}$ & 5.3 & 5.6 & 7.3 & 9.2 & +3.9 & +73.6 & 0.002 \\
\hline
\end{tabular}

${ }^{\mathrm{a}}$ Linear time trend from 2006/07 to 2012/13 estimating odds of past year cannabis use by demographic subgroup. ${ }^{\mathrm{b}} \mathrm{Chronic}$ diseases include diabetes, heart disease, stroke, sleep apnea and cirrhosis.

prevalence of $12.3 \%$ in $2011 / 12(P<0.001)$. In addition, cannabis use increased significantly throughout the entire study period among those over the age of 50 with two or more chronic conditions $(P=0.001)$, depression in the past year $(P=0.001)$ and adults who received mental health treatment $(P=0.002)$. 
Table 3 presents use characteristics and perceived risk of cannabis use among the 1919 individuals who reported past-year cannabis use in the sample of aggregated data from 2006 to 2013. Within this subsample of pastyear cannabis users, $6.9 \%$ of those met DSM-IV criteria, abuse or dependence. Most reported initiating cannabis use before the age 18 and reported either buying it or receiving it free. The frequency of reported cannabis use had two peaks: almost a third (30.8\%) reporting using 1-11 days, and almost a quarter (24.2\%) reportedly using on 100-299 days in the past year. The majority of cannabis users reported perceiving either no risk or slight risk of using cannabis monthly $(85.3 \%)$ or once or twice a week $(79.0 \%)$.

Table 3 Patterns and attitudes regarding cannabis use among past-year users aged 50 years and older, 2006-13 $(n=1919)$.

\begin{tabular}{|c|c|c|}
\hline & $\begin{array}{l}\text { Weighted } \\
\%\end{array}$ & $\begin{array}{l}\text { Unweighted } \\
n\end{array}$ \\
\hline $\begin{array}{l}\text { Meets DSM-IV criteria for cannabis } \\
\text { abuse or dependence }\end{array}$ & 6.9 & 134 \\
\hline Dependence & 3.7 & 69 \\
\hline Abuse & 3.1 & 65 \\
\hline \multicolumn{3}{|l|}{ Age of initiation of cannabis use, years } \\
\hline$<18$ & 54.2 & 1040 \\
\hline $18-35$ & 41.9 & 801 \\
\hline $36-55$ & 3.1 & 48 \\
\hline$>55$ & 0.8 & 15 \\
\hline \multicolumn{3}{|c|}{ Frequency of cannabis use in the past 12 months, in days } \\
\hline $1-11$ & 30.8 & 577 \\
\hline $12-49$ & 18.9 & 355 \\
\hline $50-99$ & 12.0 & 229 \\
\hline $100-299$ & 24.2 & 459 \\
\hline $300+$ & 14.1 & 264 \\
\hline \multicolumn{3}{|c|}{ Method of obtaining last used cannabis } \\
\hline Bought & 45.7 & 855 \\
\hline Traded for it & 1.7 & 35 \\
\hline Received free/shared & 48.0 & 934 \\
\hline Grew it & 3.2 & 60 \\
\hline Unspecified & 1.4 & 35 \\
\hline \multicolumn{3}{|l|}{ Source of last used cannabis bought } \\
\hline Friend & 74.6 & 791 \\
\hline Relative/family & 7.0 & 77 \\
\hline Someone just met/did not know & 13.2 & 129 \\
\hline Unspecified & 5.3 & 70 \\
\hline \multicolumn{3}{|c|}{ Perceived risk of cannabis use once a month } \\
\hline No risk & 46.6 & 880 \\
\hline Slight risk & 38.7 & 713 \\
\hline Moderate risk & 10.5 & 219 \\
\hline Great risk & 4.1 & 96 \\
\hline \multicolumn{3}{|c|}{ Perceived risk of cannabis use once or twice a week } \\
\hline No risk & 37.3 & 703 \\
\hline Slight risk & 41.7 & 779 \\
\hline Moderate risk & 16.1 & 312 \\
\hline Great risk & 5.0 & 110 \\
\hline
\end{tabular}

Adjusted results from the multivariable logistic regression model suggest that younger (50-64) participants (versus older; $\geq 65$ ), males (versus females), those reporting an annual household income $<\$ 20000$ (versus incomes $>$ \$20000) and those who reported being divorced or never married (versus married) had higher odds of reporting past-year cannabis use (Table 4). Pastyear use of tobacco, alcohol and illicit drugs robustly increased the odds for reporting past-year cannabis use. History of multiple chronic conditions was associated with lower odds of reporting past-year cannabis use, but those who received mental health treatment were at higher odds for reporting cannabis use. The association between cannabis use and past year anxiety or depression was no longer statistically significant after adjusting for other participant characteristics. In sensitivity analyses, we found no statistically significant interaction between time and gender or age, indicating no differential change in the relationship between cannabis use by gender or age over time. Of note, we found a significant interaction between gender and age, indicating lower odds of cannabis use among women aged 65+ compared to women aged $50-64(\mathrm{aOR}=0.66,95 \%$ confidence interval 0.45-0.96). There was no substantive change in the magnitude or direction of the other covariates in the adjusted model.

\section{DISCUSSION}

The prevalence of cannabis use among older adults is increasing; however, little is known about recent trends and correlates of use. We found a significant increase in the prevalence of past-year cannabis use in the United States among older adults from 2006/07 to 2012/13, with large relative increases particularly among those aged 65 and older. There was a significant increase in reported cannabis use among those who also reported use of tobacco, alcohol or other illicit drugs. However, the prevalence increased significantly across almost all characteristics except for race/ethnicity, with only nonHispanic whites reporting significant increases over time. Demographic correlates of past-year use of cannabis that include younger age, male, not married and low income are similar to past studies $[15,16]$, although our study also showed a large increase in use by non-Hispanic whites.

This increase in cannabis use is not unanticipated, given the high rates of substance use among the Baby Boomer generation. The trends noted in our study are probably capturing a major transition in the aging of the Baby Boomer generation, and highlights how this cohort of older adults are different in substance use behaviors compared to the generation before it. Given that the majority of Baby Boomers have still not yet reached the age 65 , 
Table 4 Multivariable logistic regression of past-year cannabis use by sociodemographic, substance use, and health characteristics among aged adults $\geq 50$ years in the 2006-13 NSDUH $(n=47140)$.

\begin{tabular}{|c|c|c|c|c|c|c|}
\hline Characteristic & OR & $(95 \% \mathrm{CI})$ & $P$-value & $a O R$ & $(95 \%$ CI) & P-value \\
\hline \multicolumn{7}{|l|}{ Year } \\
\hline 2006-07 & 1.00 & & & 1.00 & & \\
\hline 2008-09 & 1.17 & $(0.95,1.44)$ & 0.141 & 1.14 & $(0.91,1.42)$ & 0.224 \\
\hline $2010-11$ & 1.47 & $(1.22,1.78)$ & $<0.001$ & 1.53 & $(1.26,1.86)$ & $<0.001$ \\
\hline $2012-13$ & 1.72 & $(1.40,2.11)$ & $<0.001$ & 1.78 & $(1.43,2.22)$ & $<0.001$ \\
\hline \multicolumn{7}{|l|}{ Age group (years) } \\
\hline $50-64$ & 1.00 & & & 1.00 & & \\
\hline $65+$ & 0.15 & $(0.11,0.19)$ & $<0.001$ & 0.26 & $(0.20,0.34)$ & $<0.001$ \\
\hline \multicolumn{7}{|l|}{ Gender } \\
\hline Male & 1.00 & & & 1.00 & & \\
\hline Female & 0.44 & $(0.39,0.49)$ & $<0.001$ & 0.50 & $(0.44,0.57)$ & $<0.001$ \\
\hline \multicolumn{7}{|l|}{ Race/ethnicity } \\
\hline Non-Hispanic white & 1.00 & & & 1.00 & & \\
\hline Non-Hispanic black & 1.23 & $(1.00,1.52)$ & 0.047 & 0.97 & $(0.76,1.23)$ & 0.808 \\
\hline Hispanic & 0.62 & $(0.47,0.83)$ & 0.002 & 0.59 & $(0.43,0.81)$ & 0.002 \\
\hline Other & 0.72 & $(0.51,1.02)$ & 0.064 & 0.86 & $(0.60,1.23)$ & 0.395 \\
\hline \multicolumn{7}{|l|}{ Education } \\
\hline$<$ High school & 1.00 & & & 1.00 & & \\
\hline High school & 0.99 & $(0.81,1.21)$ & 0.902 & 0.84 & $(0.65,1.07)$ & 0.154 \\
\hline Some college & 1.28 & $(1.06,1.55)$ & 0.013 & 1.03 & $(0.83,1.29)$ & 0.786 \\
\hline College or more & 1.11 & $(0.91,1.35)$ & 0.318 & 1.05 & $(0.80,1.37)$ & 0.741 \\
\hline \multicolumn{7}{|l|}{ Income } \\
\hline$<\$ 20000$ & 1.00 & & & 1.00 & & \\
\hline$\$ 20000-49999$ & 0.83 & $(0.72,0.95)$ & 0.008 & 0.73 & $(0.63,0.84)$ & $<0.001$ \\
\hline$\$ 50000-74999$ & 1.00 & $(0.84,1.18)$ & 0.998 & 0.65 & $(0.53,0.79)$ & $<0.001$ \\
\hline$\geq \$ 75000$ & 1.10 & $(0.91,1.32)$ & 0.306 & 0.67 & $(0.53,0.83)$ & 0.001 \\
\hline \multicolumn{7}{|l|}{ Marital status } \\
\hline Married & 1.00 & & & 1.00 & & \\
\hline Widowed & 0.48 & $(0.36,0.63)$ & $<0.001$ & 1.08 & $(0.81,1.43)$ & 0.605 \\
\hline Divorced or separated & 2.73 & $(2.36,3.17)$ & $<0.001$ & 1.89 & $(1.62,2.22)$ & $<0.001$ \\
\hline Never married & 2.83 & $(2.32,3.46)$ & $<0.001$ & 1.78 & $(1.41,2.24)$ & $<0.001$ \\
\hline \multicolumn{7}{|l|}{ Tobacco use } \\
\hline No past year use & 1.00 & & & 1.00 & & \\
\hline Past year use & 5.38 & $(4.70,6.17)$ & $<0.001$ & 2.92 & $(2.49,3.41)$ & $<0.001$ \\
\hline \multicolumn{7}{|l|}{ Alcohol use } \\
\hline No past year use & 1.00 & & & 1.00 & & \\
\hline Past year use & 4.72 & $(3.86,5.77)$ & $<0.001$ & 3.42 & $(2.79,4.19)$ & $<0.001$ \\
\hline \multicolumn{7}{|l|}{ Illicit drug other than marijuana } \\
\hline No past-year use & 1.00 & & & 1.00 & & \\
\hline Past-year use & 15.01 & $(12.46,18.08)$ & $<0.001$ & 7.43 & $(6.07,9.10)$ & $<0.001$ \\
\hline \multicolumn{7}{|l|}{ Chronic disease $^{\mathrm{a}}$} \\
\hline Multiple chronic conditions ( $\geq 2$ chronic disease) & 0.68 & $(0.57,0.80)$ & $<0.001$ & 0.83 & $(0.69,0.99)$ & 0.040 \\
\hline \multicolumn{7}{|l|}{ Mental health } \\
\hline Depression in the past year & 2.40 & $(1.98,2.91)$ & $<0.001$ & 1.30 & $(0.94,1.81)$ & 0.109 \\
\hline Anxiety in past year & 2.29 & $(1.88,2.80)$ & $<0.001$ & 1.07 & $(0.78,1.45)$ & 0.675 \\
\hline Received mental health treatment in past year & 2.22 & $(1.96,2.52)$ & $<0.001$ & 1.44 & $(1.15,1.79)$ & 0.002 \\
\hline
\end{tabular}

${ }^{\mathrm{a}}$ Chronic diseases include diabetes, heart disease, stroke, sleep apnea and cirrhosis. $\mathrm{OR}=$ odds ratio; aOR = adjusted OR; $\mathrm{CI}=$ confidence interval .

we will probably continue to see the trends continue into the next decade. In addition, with shifting attitudes and legalization of recreational cannabis and its use for medicinal purposes, further research is needed to determine whether these changes in attitudes and/or policies affect prevalence of use among older adults, particularly in other countries.
With increased availability and use of cannabis by older adults, the benefits and risks of cannabis need to be evaluated rigorously. Alcohol use among older adults has been studied in depth, so we have an adequate understanding the specific risks $[29,30]$, potential benefits $[31,32]$, guidelines for quantifying recommended limits 
of alcohol for older adults [33] and screening and treatment [6]. However, little to no research has examined cannabis use among older adults in the same manner. While studies examining the benefits of cannabis for medicinal use is emerging, such as for chronic pain [34], the risks for older adults have not been well defined.

Aging is characterized by physiological and social changes that make older adults vulnerable to impaired function, chronic disease and geriatric conditions such as falls and cognitive impairment [35]. Therefore, the physiological effects of cannabis may lead to poor outcomes in vulnerable older adults who have multiple chronic conditions. For instance, its effects on increasing heart rate may increase the risk of cardiovascular disease, particularly among older adults who may have pre-existing disease [36]. Smoking cannabis may also lead to lung illness and infection, and older adults are more vulnerable to such conditions [36]. Cannabis has also been tied to cerebrovascular events [37] for which, again, increasing age is a risk factor. In addition, cannabis use has acute effects on cognitive function, and may have residual long-term effects $[23,38]$. A recent study that followed a cohort of adults (aged 18-30) for 25 years found that current use at follow-up was associated with impaired verbal memory and slower processing speed, while past cumulative exposure was associated with impaired verbal memory, but not with other cognitive functioning [39]. It is unclear how the effects of cannabis on cardiovascular, cognitive and pulmonary function may ultimately impact upon the chronic diseases, geriatric conditions and everyday function of older adults.

This study shows significant increases in cannabis use throughout the study period by adults with multiple chronic conditions, which has not been described previously. Therefore, an increasing number of older adults with chronic diseases appear to be especially vulnerable to the possible risks of cannabis. In addition, our study showed that the majority of older adults who reported cannabis use perceived that cannabis is associated with either no risk or only a slight risk. One particular concern is falls among older adults who use cannabis.

Unintentional falls are a common event for older adults and a major cause of morbidity and mortality linked with a decline in functional status and disability [40]. Alcohol use has been associated with falls among older adults [33], but no studies have examined the risk of falls among older cannabis users. Therefore, as further studies define more clearly the risks of cannabis for older adults, patients need to be educated about them.

Furthermore, we also describe an older population that may be at particularly high risk given their likelihood of also cigarette smoking, and/or use of alcohol or illicit drugs, which was found in an earlier study [16]. The concurrent use of multiple substances may make older adults further vulnerable to poor physical and mental health outcomes [6], and may impact upon their care. In addition, older adults are not as likely to be screened for substance use [41], often because of perceptions of older adults as being unlikely to engage in such behaviors [42]. The findings from this study highlight the imperative to screen older adults for substance use.

This study was subject to limitations. The NSDUH replies on self-report and therefore is subject to recall bias and social-desirability bias. The latter may be particularly true for alcohol or drug use where respondents may not report their substance use [43]. Also, recall bias in particular may be an issue for older adults to recall events from the distant past (for example, age of initiation of cannabis use). Secondly, because NSDUH samples the civilian, noninstitutionalized population, it does not include homeless, active-duty military, incarcerated or institutionalized adults who may have different drug patterns than those surveyed. Thirdly, because of changes to the NSDUH survey throughout the years, we were unable to examine cannabis use by metropolitan statistical area for the entire study period, limiting further examination of cannabis use by urbanicity. In addition, we were unable to assess the use of cannabis for medical purposes or as recommended by a health-care professional, as this was only introduced in the NSDUH in 2013 [26]. Therefore, we are unable to assess whether the increased use of cannabis by older adults was due to medicinal use, nor could we assess trends of cannabis use for medical purposes. Future studies will be needed to examine trends of cannabis use for medical reasons by older adults. Finally, the survey is cross-sectional, and different participants were sampled each year of the study period; therefore, this study cannot establish causality.

\section{CONCLUSIONS}

This study shows that prevalence of self-reported cannabis use in the past year is increasing among older adults in the United States. Findings add evidence to dispel the myth that older adults do not use recreational drugs; we hope the results encourage providers to screen patients of all ages for substance use. The changing landscape towards normalized and legalized cannabis throughout the world dictates further studies to understand more clearly the vulnerabilities, benefits and risks of its use by older adults, similar to how we now understand alcohol use among older adults. In particular, understanding how cannabis use may affect geriatric conditions and function in older adults with multiple chronic conditions living in different countries is an imperative. 


\section{Declaration of interests}

S.S.M. was a consultant to Purdue Pharma until December 2014 for secondary data analyses for the NESARC data on alcohol and prescription opioids, unrelated to the analyses described in this manuscript. No other disclosures are reported.

\section{References}

1. Hawken A., Caulkins J., Kilmer B., Kleiman M. Quasi-legal cannabis in Colorado and Washington: local and national implications. Addiction 2013; 108: 837-8.

2. Healy, J. Voters ease cannabis laws in 2 states, but legal questions remain. New York Times, 7 November 2012. Available at: http://www.nytimes.com/2012/11/08/us/politics/marijuana-laws-eased-in-colorado-and-washington.html (accessed 20 June 2016) (Archived at http://www. webcitation.org/6iOoh3hC0).

3. Pew Research Center. Majority now supports legalizing cannabis. Available at: http://www.people-press.org/2013/04/ 04/majority-now-supports-legalizing-marijuana/ (accessed 20 June 2016) (Archived at http://www.webcitation.org/ 6iOq1GAtA).

4. United States Census Bureau. The Older Population in the United States: 2012. Available at: http://www.census.gov/ population/projections/data/national/2012/summarytables. html (accessed 20 June 2016) (Archived at http://www. webcitation.org/6iOpwd4w5).

5. Blazer D. G., Wu L. The epidemiology of substance use and disorders among middle aged and elderly community adults: national survey on drug use and health. Am J Geriatr Psychiatry 2009; 17: 237-45.

6. Kuerbis A., Sacco P., Blazer D., Moore A. A. Substance abuse among older adults. Clin Geriatr Med 2014; 30: 629-54.

7. Moore A. A., Karno M. P., Grella C. E., Lin J. C., Warda U., Liao D. H. et al. Alcohol, tobacco, and nonmedical drug use in older U.S. adults: data from the 2001/02 National Epidemiologic Survey of Alcohol and Related Conditions. J Am Geriatr Soc 2009; 57: 2275-81.

8. Center for Behavioral Health Statistics and Quality. Behavioral health trends in the United States: results from the 2014 National Survey on Drug Use and Health (HHS Publication no. SMA 15-4927, NSDUH Series H-50). Available at: http://www.samhsa.gov/data/sites/default/files/NSDUHFRR1-2014/NSDUH-FRR1-2014.pdf (accessed 20 June 2016) (Archived at http://www.webcitation.org/6iOpQstvt).

9. United Nations Office on Drugs and Crime. World Drug Report 2015. Available at: https://www.unodc.org/documents/ wdr2015/World_Drug_Report_2015.pdf (accessed 20 June 2016) (Archived at http://www.webcitation.org/6j2oAq6x0).

10. Fahmy V., Hatch S. L., Hotopf M., Stewart R. Prevalences of illicit drug use in people aged 50 years and over from two surveys. Age Ageing 2012; 41: 553-6.

11. Hasin D. S., Saha T. D., Kerridge B. T., Goldstein R. B., Chou S. P., Zhang H. et al. Prevalence of marijuana use disorders in the United States between 2001 and 2002 and 2012-2013.JAMA Psychiatry 2015; 72: 1235-42.

12. Miech R., Koester S. Trends in U.S., past-year marijuana use from 1985 to 2009: an age-period-cohort analysis. Drug Alcohol Depend ; 2012: 259-67.

13. Agrawal A., Lynskey M. T. Correlates of later-onset cannabis use in the National Epidemiological Survey on Alcohol and
Related Conditions (NESARC). Drug Alcohol Depend 2009; 105: 71-5.

14. Satre D. D., Sterling S. A., Mackin R. S., Weisner C. Patterns of alcohol and drug use among depressed older adults seeking outpatient psychiatric services. Am J Geriatr Psychiatry 2011; 19: 695-703.

15. Colliver J. D., Compton W. M., Gfroerer J. C., Condon T. Projecting drug use among aging baby boomers in 2020. Ann Epidemiol 2006; 16: 257-65.

16. DiNitto D. M., Choi N. G. Marijuana use among older adults in the U.S.A.: user characteristics, patterns of use, and implications for intervention. Int Psychogeriatr 2010; 23: 732-41.

17. Choi N. G., DiNitto D. M., Marti C. N. Older-adult marijuana users and ex-users: Comparisons of sociodemographic characteristics and mental and substance use disorders. Drug Alcohol Depend 2016; 165: 94-102.

18. Gallup. In U.S., $58 \%$ Back Legal Marijuana Use. Available at: http://www.gallup.com/poll/186260/back-legal-marijuana. aspx (accessed 20 June 2016) (Archived at http://www. webcitation.org/6iOpUm9h0).

19. Palamar J. J. An examination of opinions toward marijuana policies among high school seniors in the United States. J Psychoact Drugs 2014; 46: 351-61.

20. Swift, A. For first time, Americans favor legalizing marijuana: Support surged 10 percentage points in past year, to $58 \%$. Available at: http://www.gallup.com/poll/165539/first-timeamericans-favor-legalizing-marijuana.aspx (accessed 20 June 2016) (Archived at http://www.webcitation.org/6iOpoOU01).

21. Bonn-Miller M. O., Babson K. A., Vandrey R. Using cannabis to help you sleep: heightened frequency of medical cannabis use among those with PTSD. Drug Alcohol Depend 2014; 136: 162-5.

22. Walsh Z., Callaway R., Belle-Isle L., Capler R., Kay R., Lucas P. et al. Cannabis for therapeutic purposes: patient characteristics, access, and reasons for use. Int J Drug Policy 2013; 24: 511-16.

23. Hazekamp A., Ware M. A., Muller-Vahl K. R., Abrams D., Grotenhermen F. The medicinal use of cannabis and cannabinoids - an international cross-sectional survey on administration forms. J Psychoact Drugs 2013; 45: 199-210.

24. Meier M. H., Caspi A., Ambler A., Harrington H., Houts R., Keefe R. S. E. et al. Persistent cannabis users show neuropsychological decline from childhood to midlife. Proc Natl Acad Sci USA 2012; 109: E2657-64.

25. Schweinsburg A. D., Brown S. A., Tapert S. F. The influence of marijuana use on neurocognitive functioning in adolescents. Curr Drug Abuse Rev 2008; 1: 99-111.

26. Substance Abuse and Mental Health Services Administration (SAMHSA). Results from the 2012 National Survey on Drug Use and Health: Summary of National Findings, NSDUH Series H46, HHS Publication no. (SMA) 13-4795. Rockville, MD: Substance Abuse and Mental Health Services Administration; 2013.

27. Jones C. M., Logan J., Gladden R. M., Bohm M. K. Vital signs: demographic and substance use trends among heroin usersUnited States, 2002-2013. Morb Mortal Wkly Rep 2015; 64: 719-25.

28. Heeringa S., West B. T., Berglund P. A. Applied Survey Data Analysis. Boca Raton, FL: Chapman \& Hall/CRC; 2010.

29. Kennedy G. J., Efremova I., Frazier A., Saba A. The emerging problems of alcohol and substance abuse in late life. J Soc Distress Homel 1999; 8: 227-39.

30. Moore A. A., Endo J. O., Carter M. K. Is there a functional relationship between excessive drinking and functional impairment in older persons? J Am Geriatr Soc 2003; 51: $44-9$. 
31. Chen L. Y., Hardy C. L. Alcohol consumption and health status in older adults. J Aging Health 2009; 21: 824-47.

32. Mukamal K. J., Kuller L. H., Fitzpatrick A. L., Longstreth W. T., Mittleman M. A., Siscovick D. S. Prospective study of alcohol consumption and risk of dementia in older adults. JAMA 2003; 289: 1405-13.

33. National Institute on Aging. Alcohol Use in Older People. Available at: https://www.nia.nih.gov/health/publication/alcohol-use-older-people (accessed 25 November 2016) (Archived at http://www.webcitation.org/6mHk1HoMS on 25 November 2016).

34. Deshpande A., Mailis-Gagnon A., Zoheiry N., Lakha S. F. Efficacy and adverse effects of medical marijuana for chronic noncancer pain: systematic review of randomized controlled trials. Cam Fam Physician 2015; 61: e372-81.

35. Cigolle C. T., Langa K. M., Kabeto M. U., Tian Z., Blaum C. S. Geriatric conditions and disability: the health and retirement study. Ann Intern Med 2007; 147: 156-64.

36. National Institute on Drug Abuse. DrugFacts: Marijuana. Available at: http://www.drugabuse.gov/publications/ drugfacts/marijuana (accessed 20 June 2016) (Archived at http://www.webcitation.org/6iOpe5wLg).
37. Hackam D. G. Cannabis and stroke. Stroke 2015; 46: 852-6.

38. Grant L., Gonzalez R., Carey C. L., Natarajan L., Wolfson T. Non-acute (residual) neurocognitive effects of cannabis use: a meta-analytic study. J Int Neuropsychol Soc 2003; 9: 679-89.

39. Auer R., Vittinghoff E., Yaffe K., Kunzi A., Kertesz S. G., Levine D. A. et al. Association between lifetime marijuana use and cognitive function in middle age: the Coronary Artery Risk Development in Young Adults (CARDIA) study. JAMA Intern Med 2016; 176: 352-61.

40. Tinetti M. E., Williams C. S. The effect of falls and fall injuries on functioning in community-dwelling older persons. $J$ Gerontol A Biol Sci Med Sci 1998; 53: M112-9.

41. Duru O. K., Xu H., Tseng C. H., Mirkin M., Ang A., Tallen L. et al. Correlates of alcohol-related discussions between older adults and their physicians. J Am Geriatr Soc 2010; 58: 2369-74.

42. Dar K. Alcohol use disorders in elderly people: fact or fiction? Adv Psychiatr Treat 2006; 12: 173-81.

43. Tourangeau R., Smith T. W. Asking sensitive questions - the impact of data collection mode, question format, and question context. Public Opin Q 1996; 60: 275-304. 